\title{
Emerging threat in antifungal resistance on superficial dermatophyte infection
}

\author{
R Sultana ${ }^{1}$, M Wahiduzzaman ${ }^{2}$
}

\begin{abstract}
Background: Dermatophytosis are most common fungul infection globally and according to WHO the prevalence is about 20-25\% and does not spare people of any race or age. Over the past few years antifungal resistance has been emerged due to irrational use of antifungal drugs in cutaneous mycosis.
\end{abstract}

Objective: The aim of the study is to evaluate the efficacy of different antifungul drugs (Terbinafin. Fluconazole, Itraconazole, Griseofulvin) on superficial mycosis depending on various factors.

Methods: This prospective study was conducted among the Superficial fungul infected patients from April' 2017 to October 2017 in Khulna Medical College Hospital (KMCH) and dermatologist's private chamber in Khulna city. All the enrolled patients were put on oral Terbinafin, Fluconazole, Itraconazole and Griseofulvin. Each patient was given single antifungal drug orally. These cases were thus followed up after two months of treatment to look for persistent infection, cure or any relapse clinically.

Result: Among 194 patient 89 were given Tab. Terbinafin (250mg) where resistance cases were 20.22\%. More cases (33.96\%) were resistant to Cap. Fluconazol (50mg). High percentage of cases were resistant to Cap. Itraconazole (76.47\%). Griseofulvin resistant cases were observed in 25.71\%. Drug response is very poor (69\%) in patient who had been suffering from diabetes mellitus.

Conclusion: Appropriate antifungal drugs should be chosen with strict indication, dose, duration, selection of perfect local preparation and taking laboratory facilities where necessary.

Keywords : Dermatophytosis, Antifungal resistance, Public health.

\section{Introduction}

Dermatophytosis (Superficial fungus infection) are the most common infective dermatoses, seen globally. According to WHO the prevalence rate of superficial mycosis has been found to be $20 \%$ $25 \%{ }^{1}$ It is the most common public health problem in hot $\&$ humid climate of tropical and sub-tropical counties like Bangladesh. Mycosis may have significant negative social, psychological and occupational impact on health. The pathogenesis of chronic or recurrent dermatophytic infection are not well understood. It is suggested that patients with immunocompromised status, Diabete Mellitus, atopy and use of corticosteroid are the predisposed factors for recurrent infection. It is an infection caused by three genera Trichophyton, Epidermophyton and Microsporum. Various oral and topical agents have shown efficacy against these genera of dermatophytes. The commonly used drugs are Allylamine (Terbinafin, Natifine), amongst Azoles the triazole group (Fluconazole, Itraconazole, Terconazole) and griseofulvin. However, recently it is observed that decreased efficacy of these drugs in patients are quite common with tinea infection, which has never been encountered previously. Although the prevalence of drug resistance in fungi is below that observed in bacteria, mycologists; now believe that selective pressure will, over time, lead to more widespread resistance. ${ }^{2}$ The principal reason may include low plasma concentration incomplete mycological cure which are very common following therapy.3,4 The aim of the study is to review and evaluate the efficacy of different antifungul drugs on superficial mycosis depending on various other factors. In Bangladesh no study has been conducted in this field till now.

1. Razia Sultana DDV, Associate Professor, Department of Dermatology, Khulna Medical College (E-mail: raziaprof@yahoo.com)

2. Md Wahiduzzaman DDV, Assistant Professor, Department of Dermatology, Khulna Medical College 


\section{Material and methods}

This prospective study was conducted in skin and VD-OPD in Khulna Medical college hospital, Khulna and different private dermatologist's chambers in Khulna city for 07 months from April 2017 to October 2017 in hot and humid weather. A total of 234 (male \& female) patients clinically and mycologically (where necessary) diagnosed to have superficial fungal infection such as tinea corporis, tinea cruris, tinea facie, tinea manum, tinea pedis were included in this study. Patients were given special attention for occupations, duration of disease, history of previous management for this disease and associated diseases such as diabetes mellitus (DM) or immunocompromised.

All the enrolled patients were put on oral Terbinafin, Fluconazole, Itraconazole and Griseofulvin. Each patient was given single antifungal drug orally: Tablet Terbinafin (250 mg) was given once daily for one month or Capsule Fluconazole $(50 \mathrm{mg}$ ) once daily for six weeks or Capsule Itraconazole (100 mg) twice daily for one month or tablet Griseofulvin (500 mg) once daily for six weeks.

Clinical cure was taken as the absence of itching (local area), visible erythema, and scaling. Residual pigmentation was present in some cases but was not taken as suggestive of incomplete cure. These cases were thus followed up after two months of treatment to look for persistent infection, cure or any relapse clinically. Ultimately, at the end of two months only 194 patients were followed up and 40 patients were dropped out from the study. The data after collection was compiled and compared. Statistical test was conducted to compare the difference between these drugs and $\mathrm{P}<0.05$ was considered as significant.

\section{Result}

Among 194 patient 55 were male and 137 were female and male female ratio were 0.4:1. Mean age of population was 34.86. In this study, tinea corporis was the most common infection (51.03\%). The combined infection, T. Corporis with T. Cruris was the next most common infection and it was $16.49 \%$. The least common infection was the $\mathrm{T}$. Facie $(2.06 \%)$. (Table I).
Table I

Demographic characters of patients.

\begin{tabular}{lc}
\hline & No of patients (\%) \\
\hline Age Range (mean) Yrs. & 34.86 \\
Gender & $55(28.35)$ \\
Male & $137(70.61)$ \\
Female & \\
Type of Dermatophytosis & \\
Common infection & $99(51.03)$ \\
T. Corporis & $29(14.94)$ \\
T. Cruris & $4(2.06)$ \\
T. Faciei \& barbi & $7(3.60)$ \\
T. Pedis & \\
Combined infection & $32(16.40)$ \\
T. Corporis + T. Cruris & $23(11.85)$ \\
T. Faciei+T. Corporis+T. Cruris & \\
\hline
\end{tabular}

Table II revealed that 89 patients were given Tab Terbinafin where resistance cases were $20.22 \%$. Amongst 53 cases, $33.96 \%$ were resistant to Cap Fluconazole. High percentage of cases were resistant to Cap Itraconazole (76.47\%), Tab Grisefulvin resistant cases were observed in $25.71 \%$. A grand total resistant case with these different drugs showed $28.86 \%$.

Table II

Comparative efficacy of different drugs

\begin{tabular}{lccc}
$\begin{array}{l}\text { Drug and dosage } \\
\text { No of pt }\end{array}$ & $\begin{array}{c}\text { Crued } \\
(\%)\end{array}$ & $\begin{array}{c}\text { Not cured } \\
(\%)\end{array}$ \\
\hline $\begin{array}{l}\text { Tab. Terbinafin } \\
\text { (250mg once) }\end{array}$ & 89 & $71(79.77)$ & $18(20.22)$ \\
$\begin{array}{l}\text { Cap. Fluconazole } \\
\text { (50mg once) }\end{array}$ & 53 & $35(66.03)$ & $18(33.96)$ \\
$\begin{array}{l}\text { Cap. Itraconazole } \\
\text { (100mg twice) }\end{array}$ & 17 & $4(23.52)$ & $13(76.47)$ \\
$\begin{array}{l}\text { Tab. Griseofulvin } \\
\text { (500mg once) }\end{array}$ & 35 & $26(74.28)$ & $09(25.71)$ \\
\hline Total & 194 & 136 & $58(28.86)$ \\
\hline
\end{tabular}

In patients suffering from DM the drug response was very poor and about $69 \%$ patients showed no clinical cure. This was nearly same for AIDS patient (table III). Table IV showed patient who had used topical steroid or steroid mixed antifungal agent just prior to therapeutic trial which obscure the healing. 
Table III

Associated disease with drug response.

\begin{tabular}{lccc}
\hline Disease & No of pt & $\begin{array}{c}\text { Crued } \\
(\%)\end{array}$ & $\begin{array}{c}\text { Not cured } \\
(\%)\end{array}$ \\
\hline DM & 13 & $4(30.76)$ & $9(69)$ \\
Psoriasis & 5 & 0 & 0 \\
Hepatitis & 1 & 0 & $1(100)$ \\
AIDS & 2 & 0 & $2(100)$ \\
\hline
\end{tabular}

Table IV

Response to the use of topical steroid

\begin{tabular}{lrcc}
\hline Topical Agent & $\begin{array}{c}\text { No of pt Crued } \\
(\%)\end{array}$ & $\begin{array}{c}\text { Not cured } \\
(\%)\end{array}$ \\
\hline Topical steroid & 15 & $1(6.6)$ & $14(93.33)$ \\
Steroid mixed antifungal 36 & $12(33.33)$ & $24(66.66)$ \\
\hline
\end{tabular}

\section{Discussion}

Dermatophytosis, being the most common dermatologic condition 5 does not spare people of any race or age. ${ }^{6}$ The antifungal drugs in the therapy of fungal diseases can lead to the development of antifungal resistance. Clinical resistance has been defined as persistence or progression of an infection despite appropriate antifungal therapy. Over the past few years, antifungal resistance has been emerged due to irrational use of antifungal agents in cutaneous mucosis. ${ }^{7}$ Same is true for our study. Self administration of antifungal. drugs over the counter or prescribed by the quack and suboptimal dose and incomplete course or substandard quality of drug is a common scenario.

In this present study, the allylamin antifungal (Terbinafin) resistance is nearly comparable to Indian study and triazole antifungal (Fluconazole, Itraconazole) resistance is more or less similar to Dominique and Pramod. ${ }^{8-10}$ The extent of the problem is variable and depends on the type of fungus, the type of antifungal agents and geographical location of hospital or study area.9 Mukharjee et al in 2003 first confirmed, the report of Terbinafin resistance in superficial fungal infection. ${ }^{11}$ Majid et al in their study reported that at the end of 12 weeks there were only $43 \%$ enrolled cases which were able to maintain a long term clinical cure after 2 weeks of oral terbinafin 4 but our study showed that in $20.22 \%$ cases there was incomple cure and relapse after 2 months of completion of treatment for 01 month. The prefered treatment for superficial dermatophytes, is Griseofulvin although some resistance has developed to oral Griseofulvin 12 similar to our study.

Drug resistance gene and genome mutation have been identified.9 Although genetic study gives the highest conclusion and rarely available in our country.

Some risk factors have been associated with superficial fungal infection like genetic factor. Poor hygiene, some diseases such as DM and immunodeficiency also negatively affect the healing process of antifungal drugs. ${ }^{13}$ The current study reveals that $69 \%$ Diabetes patient showed poor response or relapse of the disease after completion of treatment. So good control of DM is essential for treatment of the fungal disease. Frequently clinicians of different discipline prescribe combination (Steroid with antifungal) of topical agents. About $66.66 \%$ cases developed resistance amongst the 36 patient who used steroid mixed fungicidal topical preparation just prior to this therapy. This is due to reduced number of monocytes at the site of inflammation after use of topical corticosteroid. This causes decrease fungicidal activity and clearance of opsonized particle. ${ }^{14}$

The study was done with few drugs depending on clinical ground. There is limitation of this study as we did not observe the effectiveness of the drugs by culture and sensitivity due to financial constraint of the patients.

\section{Conclusion}

All doctors from different discipline should choose appropriate antifungal drugs with strict indication, dose, duration, selection of perfect local preparation, and should take laboratory facilities where necessary and advice the patient to maintain good personal hygiene. Quality control policy of the these drugs should be reviewed as well.

\section{Reference}

1. Havlickova B, Czaika VA, Friedrich M. Epidemiological trends in the skin mycosis worldwide. 2008; 4: 2-15.

2. Stephenson J. Investigators seeking new ways to stem rising tide of resistant fungi. Jour Amer Med Assoc. 2003; 277: 5-6. 
3. Hosseini M, Mclachlan AJ. Physiologically based pharmacokinetic model for terbinafine in rats and humans. Antimicrobe Agents Chemother 2002; 46: $2219-28$.

4. Majid I, Sheikh G, Kanth F, Hakak R. Relapse after oral terbinafine therapy in dermatophytosis: Clinical and mycological study. Indian $\mathrm{J}$ Dermatol 2016; 1: 529-33.

5. Gupta AK, Chaudhury M, Elewski B. TineaCorporis, tineacruris, tineanigra and piedra. Dermatolclin. 2003; 21395-400.

6. Straten MRV, Hossain MA, Ghannoum MA. Cutaneous infections dermatophytosis, onycomycosis and tineaversicolor. Infect Dis Clin North Am. 2003; 17:87-112.

7. Piyush B, Borkhatariya. Resistence in fungal infection. Dermatalk.2016; 11: 45-45.

8. Babu PR, Pravin AJS, Desmukh G, Dhoot D, Samant A, Kotak B. Efficacy and safety of terbinafin $500 \mathrm{mg}$ once daily in patients with Dermatophytosis. Indan J Dermatol 2017; 62; 395-9.
9. Sanglard D. Emerging threats in antifungal Resistant Fungal pathogen Frontiers in Medicine 2016; 3: 11 .

10. Nigam PK, Antifungal drugs and resistance: Current concepts our personal online 2015 ; 6: 212-221.

11. Mukherjee PK, Leidich SD, Islam Lither NI, Ryder NS, Ghanoum MA, Clinical trichophylonrubrumstrainekhibiting primary resistance to terbinafin Antimicrob Agents chemotherapy 2003; 47: 82-6.

12. Wingfield AB, Fernam. Obregon AC, wignal FS, green DL. Treatment of tinea imbricate; a randomized clinical trial using greseofulvin terbinafin, itroconazole and fluconazol. $\mathrm{Br} \mathrm{J}$ Dermatol. 2004; 150: 119-26.

13. Seebacher C, Bouchara JP, Mignon B. Updates on the epidermiolog of dermatophyte, infections. Mycopathologia. 2008; 166: 33552.

14. Stephen E. Wolverton. Comprehensive Dermatologic Drug therapy, 2nd ed. Philadelphia: Elsevier; 2007. p 600. 